\title{
MAGNETIC MEASUREMENTS OF THE PROTOTYPE DIPOLE FOR THE IR-FEL AT THE THOMAS JEFFERSON NATIONAL ACCELERATOR FACILITY
}

\author{
J. Karn, G. Biallas, A. Guerra, and L. Harwood \\ Thomas Jefferson National Accelerator Facility \\ 12000 Jefferson Avenue, Newport News, VA 23606 USA
}

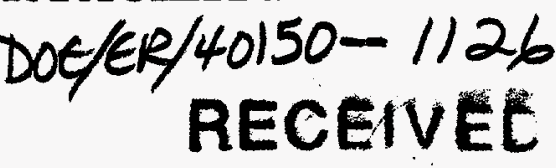

JUN 171997

Abstract

Magnetic measurements have been performed on the prototype dipole for the high power IR-FEL presently under construction at the Thomas Jefferson National Accelerator Facility. The optics-driven requirements for these magnets include low fields, large horizontal apertures, tight field homogeneity, absolute setability of core field \& integrated field, and control of the horizontal \& vertical focusing terms designed into the magnets. A prototype dipole was fabricated and underwent several iterations of mechanical adjustment and magnetic measurement. Measurements were made to quantify the effects of field clamps on vertical focusing terms and effective length. Additional tests were made using various applications of Purcell gaps and high permeability materials in order to achieve the required homogeneity. Results from the prototype have been integrated into the design of the seven families of dipoles needed for the FEL.

\section{INTRODUCTION}

A $1 \mathrm{~kW}$ infrared free-electron laser is under construction at the Thomas Jefferson National Accelerator Facility. The injector for the accelerator produces a $10 \mathrm{MeV}, 5 \mathrm{~mA} \mathrm{CW}$ electron beam. The injected beam is accelerated to $42 \mathrm{MeV}$ using a single CEBAF-style SRF cryomodule. A wiggler and optical cavity produce light in the 3 to 6.6 micron range. A transport arc recirculates the beam exiting the wiggler back through the cryomodule for energy recovery. A potential upgrade to a beam energy of $75 \mathrm{MeV}$ would produce light in the 1 micron range.

\section{DIPOLE DESIGN REQUIREMENTS}

The lattice design of the IR-FEL was tightly constrained due to the low energy, high current, and the $5 \%$ relative momentum spread of the recirculated beam [1]. The accelerator requires a total of 29 bending dipoles consisting of 7 different families. The recirculation dipoles will be engineered for beam energies from 33 to $75 \mathrm{MeV}$. Injector dipoles will cover injection beam energies from 8 to $12 \mathrm{MeV}$. Table 1 summarizes the 7 families and their field strength requirements.

\begin{tabular}{|c|c|c|c|}
\hline $\begin{array}{c}\text { Dipole } \\
\text { Family }\end{array}$ & Quantity & $\begin{array}{c}\text { Core Field } \\
\text { Range (kG) }\end{array}$ & $\begin{array}{c}\text { Effective } \\
\text { Length (m.) }\end{array}$ \\
\hline DU & 7 & $0.45-0.67$ & 0.21 \\
\hline DV & 2 & $0.45-0.67$ & 0.43 \\
\hline DX & 4 & $1.10-2.64$ & 0.51 \\
\hline DQ & 4 & $1.10-2.64$ & 0.51 \\
\hline DW & 8 & $1.04-2.48$ & 0.41 \\
\hline DY & 2 & $1.10-2.64$ & 3.14 \\
\hline DZ & 2 & $0.92-2.20$ & 0.21 \\
\hline
\end{tabular}

Table 1: Dipole strength specifications

In the designed lattice, the dipoles are also used to manage dispersion and the beam envelope in the recirculation arc. To provide horizontal focusing four of the dipole families have wedge shaped cores with included angles ranging from $7.7^{\circ}$ to $20.0^{\circ}$. Important to vertical focusing is the end-field roll-off integral defined as:

$$
K_{1}=\int_{-\infty}^{\infty} \frac{B_{y}(z)\left[B_{0}-B_{y}(z)\right]}{g B_{0}^{2}} d z
$$

where $g$ is the height of the gap and $B_{0}$ the field strength in the center of the magnet.

The specifications for the horizontal and focusing terms are listed in Table 2 [2].

\begin{tabular}{|l|c|}
\hline \multicolumn{1}{|c|}{ Constraint } & Tolerance \\
\hline End-field roll-off integral (K1) & $0.27 \pm 0.05$ \\
\hline Field integral slope tolerance & $6.25 \times 10^{-4} / \mathrm{m} \times \mathrm{BP}$ \\
\hline Relative field integral flatness & $1 \times 10^{-4}$ \\
\hline
\end{tabular}

Table 2: Dipole focusing specifications (rms)

Excitation errors in the dipoles could lead to undesirable steering and focusing effects. This puts constraints on the reproducibility of the magnets and the knowledge of the absolute core field and integrated field. Since several of the dipole families are powered in series by a common power supply, the variance of magnets within a family is important. Excitation tolerances are listed in Table 3 [2]. 


\section{DISCLAIMER}

This report was prepared as an account of work sponsored by an agency of the United States Government. Neither the United States Government nor any agency thereof, nor any of their employees, make any warranty, express or implied, or assumes any legal liability or responsibility for the accuracy, completeness, or usefulness of any information, apparatus, product, or process disclosed, or represents that its use would not infringe privately owned rights. Reference herein to any specific commercial product, process, or service by trade name, trademark, manufacturer, or otherwise does not necessarily constitute or imply its endorsement, recommendation, or favoring by the United States Government or any agency thereof. The views and opinions of authors expressed herein do not necessarily state or reflect those of the United States Government or any agency thereof. 


\section{DISCLAMMER}

Portions of this document may be illegible in electronic image products. Images are produced from the best available original document. 


\begin{tabular}{|l|c|}
\hline \multicolumn{1}{|c|}{ Constraint } & Tolerance \\
\hline Reproducibility of integrated field & $1 \times 10^{-4}$ \\
\hline Accuracy of core field & $1 \times 10^{-3}$ \\
\hline Accuracy of integrated field & $1 \times 10^{-3}$ \\
\hline Tracking within dipole family & $1 \times 10^{-3}$ \\
\hline
\end{tabular}

Table 3: Dipole excitation error tolerances (rms)

\section{CONSTRUCTION OF PROTOTYPE DIPOLE}

A single prototype dipole (Figure 1) was fabricated to help quantify the above mentioned tolerances for all seven dipole families. The magnet was a window frame design with a AISI 1006 low-carbon steel core and two saddle-style coils. The coils were 27 turns each, wound from 0.25 inch hollow-core copper conductor, and encased in glass epoxy.

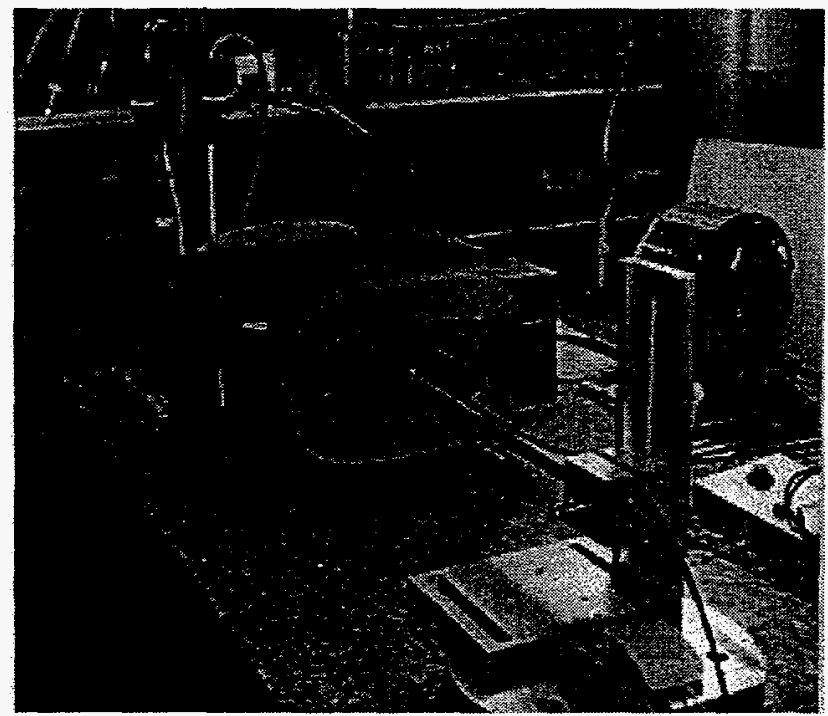

Figure 1: Prototype Dipole

\section{MAGNETIC MEASUREMENTS}

\subsection{Measurement Setup}

The measurements described below were performed using a Group 3 Hall Effect Probe and a Metrolab NMR Teslameter. The probes were attached to a cart that provided transverse and longitudinal motion. The cart referenced off a granite surface plate to maintain a constant probe orientation with the magnet. A PC-based data acquisition system recorded the probe values at the selected position steps.

\subsection{Longitudinal Measurements}

There were three areas of interest to study from longitudinal profile measurements: fringe fields, effective length, and the field roll-off integral. The extent of the fringe field was important due to the compact design of the lattice and the close proximity of the dipoles to other beamline elements. Determining the effective length to an accuracy better than $1 \times 10^{-3}$ was needed to meet the core field and field integral tolerances for all dipole families. Determination of the field roll-off integral was required to finalize the lattice design. (The lattice designer graciously left this value flexible to accommodate magnet design.)

Once fringe fields could be brought to an acceptable level, measurements could be made to quantify the effective length and the field roll-off integral. The first measurements revealed the field dropped of to $0.1 \%$ at a distance of $37.0 \mathrm{~cm}$ from the end of the iron core. To minimize the extent of fringe fields, various designs of field clamps were tested. The final design was a clamp of 0.5 inch thick steel surrounding the coil and connected to the iron core. This setup resulted in the field dropping to $0.1 \%$ at a distance of $15.0 \mathrm{~cm}$ from the iron core. Figure 2 shows the change in field fall-off with the addition of the field clamp.

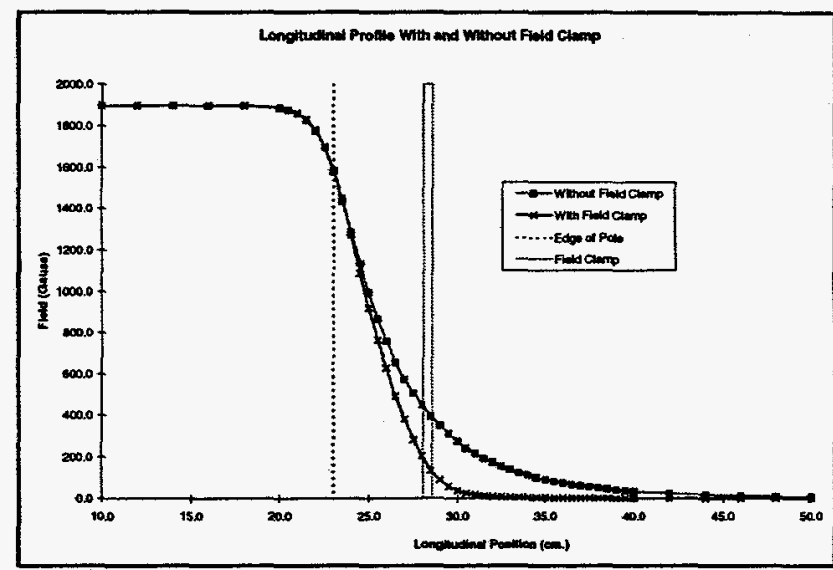

Figure 2: Longitudinal field fall-off with added field clamp.

With the design of the field clamp finalized, measurements were taken to determine the effective length and the field roll-off integral. Longitudinal profiles were made over the operating ranges needed for the 7 families of dipoles. The effective length was calculated to be $25.396 \pm 0.004 \mathrm{~cm}$. $\left(1.6 \times 10^{4}\right.$ relative $)$ over the operating range. The field roll-off integral was calculated to be $0.31 \pm 0.01$ over the operating range. Both values were within the tolerances defined in Section 2.

\subsection{Transverse Measurements}

Measurements were made to study the transverse field homogeneity inside the core. These measurements would be the first step in achieving the $1 \times 10^{-4}$ integrated field flatness defined in Section 2. The first measurements revealed a relative deviation from flatness of $\pm 1 \times 10^{-3}$, an order of magnitude larger than the requirements. The 
transverse profile is plotted in Figure 3 at several core fields.

To improve field flatness, the dipole was modified by the addition of Purcell gap plates. This consisted of adding a $1.5 \mathrm{~mm}$. plate of high permeability material (Vacoperm 70 - mumetal) to each pole, but separated from the iron by a $1.6 \mathrm{~mm}$. plate of aluminum. The return legs of the core were shimmed with iron in order to maintain the 2 inch mechanical gap. Measurements with the Purcell gap showed a relative deviation from flatness of $\pm 1 \times 10^{4}$, a drastic improvement in field homogeneity (Figure 4.)

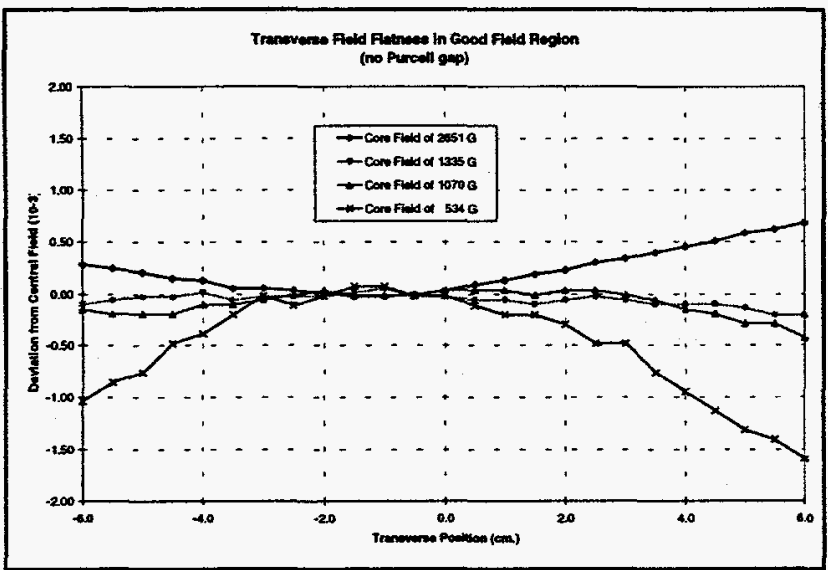

Figure 3: Relative Transverse profile at various core

fields before addition of Purcell gap plates.

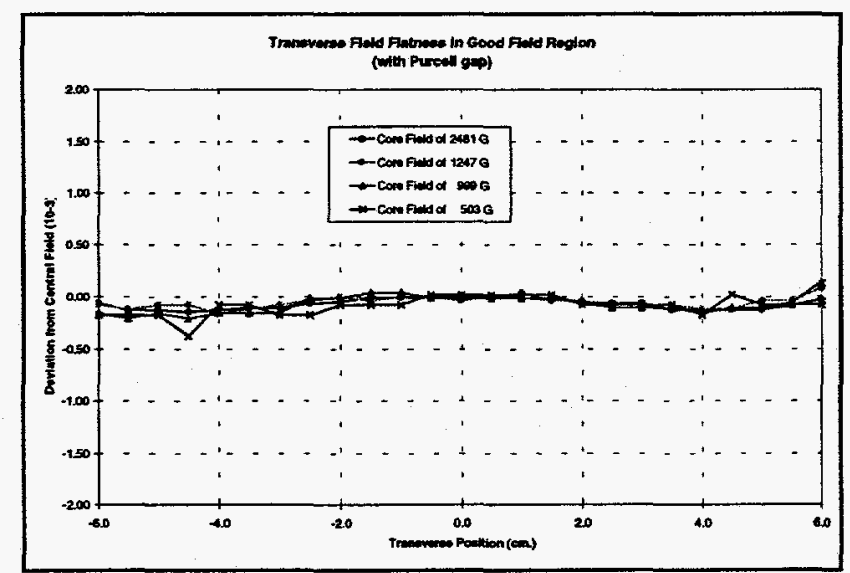

Figure 4: Relative Transverse profile at various core

fields with the addition of Purcell gap plates.

\section{DESIGNING THE PRODUCTION DIPOLES}

The seven families of dipoles vary in their aperture, effective length, and the width of their good field region. Extrapolations were made from the experimental data obtained from the prototype to the design of the production magnets. The effective length value obtained from the prototype was used to obtain a scaling factor for changes in aperture. This resulted in:

$$
\text { (Effective Length })=(\text { Core length })+(0.995)(\text { aperture })
$$

Similarly, measurements on the prototype with the Purcell gap plates showed the good field region started at a distance of $2.3 \mathrm{~cm}$. from the coils. This offset was scaled by the aperture of the production dipoles and the required core widths calculated to achieve the desired good field regions.

\section{PRODUCTION MEASUREMENT PLANS}

At the writing of this paper, several of the dipole families were being fabricated at outside vendors and the remaining families were in final design. All dipoles will undergo magnetic measurement before being installed in the accelerator enclosure. The core field, field integral, and field roll-off integral will be determined using the Hall and NMR probe stand described in Section 4.1.

To determine the transverse integrated field profile and the variation in field integral within a family, a specialized stand is under construction. This stand will use the same technique, and share common hardware, with the setup used to measure the CEBAF dipoles [3]. This method uses two pickup coils that are driven simultaneously across the aperture of two magnets of a given family. The induced signals are bucked against each other and thus measure the difference between the two magnets. This method produced a precision of $1 \times 10^{-}$ ${ }^{s}$ in measuring the relative transverse profile of the CEBAF arc dipoles [4].

\section{CONCLUSIONS}

The optics requirements of the IR-FEL accelerator result in tight tolerances on the design and measurement of the seven families of main bending dipoles. A prototype was built and went through several iterations of mechanical adjustments and magnetic measurements. The final measurements met the required specifications and were integrated into the design of the production dipoles. Design and fabrication of the production dipoles is almost complete and plans are underway for their eventual magnetic mapping.

\section{ACKNOWLEDGMENTS}

This work was supported by the U.S. DOE under contract number DE-AC05-84ER40150.

\section{REFERENCES}

[1] D. R. Douglas, Lattice Design for a High-Power Infrared FEL, these proceedings.

[2] D. R. Douglas, Error Estimates for the IR FEL Transport System, Jefferson Lab Tech Note \# 96$035,1996$.

[3] L. Harwood, Beam Transport Magnet Measurement, Jefferson Lab Tech Note \# 187, 1989.

[4] L. Harwood and J. Karn, Measured Field Performance of CEBAF Beam transport Magnets, Proceeding of Vancouver Magnet Conference, Vancouver, British Columbia, Canada, 1993. 